\title{
New Approach For Ultra-Stable TEM-Column Support Frame
}

\author{
Erik Essers, Gerd Benner, Alexander Orchowski ${ }^{*}$, Roland Kappel ${ }^{\#}$, Michael Trunz ${ }^{\text {\& }}$ \\ * LEO Elektronenmikroskopie GmbH, D-73446 Oberkochen, Germany \\ \# IDE Integrated Dynamics Engineering, D-65479 Raunheim, Germany \\ ${ }^{\&}$ IST Ingenieurbüro für Strukturmechanik Trunz, D-73408 Aalen, Germany
}

We present a new approach for an ultra-stable TEM-column support frame. The described concept is

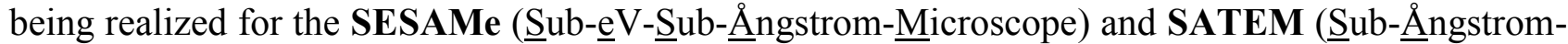
Transmission-Electron-Microscope) instruments with a specified resolution of $<0.9 \AA$, which are currently under development at LEO.

In order to reach the theoretical performance limits of future high-end TEM instruments, ultimate stable operating conditions of the electron optical setup are required. This concerns on one hand the stability of the parameters to be supplied for operation, like accelerating voltage and lens currents. On the other hand, the microscope environment itself must not alter the optical conditions. AC-stray fields and mechanical instabilities caused by building vibrations, acoustic noise or temperature changes have to be kept to a minimum. Besides minimizing all sources of disturbances, a high robustness of the electron optical column against environmental influences of this kind is required. The electron column itself, stretching from electron gun to the final detection plane, has to be optimized regarding insensitivity to changing power consumption (changing lens currents at different magnifications in either imaging or diffraction mode cause changing thermal load) and in order to obtain adequate stiffness to avoid excitation of vibrational eigenmodes.

The mechanical mount of the TEM-column onto a supporting frame defines the mechanical coupling of the optical setup to the environment. We have investigated the performance of traditional concepts for supporting TEM-columns - i.e. "standing columns", usually supported beneath the viewing chamber - and identified superior concepts regarding mechanical properties of the overall system.

Following goals were to be met: • TEM-column mounted as insensitive as possible with respect to mechanical excitations at the instruments footpoint; • Minimized deformations eventually caused by external forces transferred via the bearings between support structure and column.

The properties of several components were identified to be investigated for optimization:

- Coupling to the floor; $\bullet$ Dynamical properties of the frame; $\bullet$ Vibrational isolation between frame and column; • Coupling between bearing points and column via carrier structure; • Dynamical properties of the column.

The identified ideal solution is a "hanging column"-approach, by supporting the column close to the centre of gravity, slightly beneath the objective lens, like a pendulum. Thus the frame structure has to provide bearing points with adequate load capacity in about $1.8 \mathrm{~m}$ above floor level, resulting in a high mass. In order to limit the weight some kind of light construction is required. Furthermore the design must ensure adequate free space around the column for maximum instrument layout (including space needed for installation and deinstallation of periphery - like aperture holders, detectors, etc.). Additionally the user needs to be able to comfortably access all operational parts on the column while operating the instrument. 
For minimization of transfer of vibrations from the floor to the column, the lowest Eigenfrequency of the support frame has to be as high as achievable. On the other hand, the Eigenfrequency of the column itself should be even higher and "detuned" with respect to the frame's frequency.

Our approach is to use a box-like structure based on a triangular cross section, formed by steelplates with moderate thicknesses (between 5 and $20 \mathrm{~mm}$ ). By subsequent optimization we developed a frame model meeting the required specifications. Extended finite element model calculations reveal, that Eigenfrequencies with favourable values can be obtained: the first Eigenmode of the frame is found to be clearly above $80 \mathrm{~Hz}$ and corresponds to a torsion mode around the vertical direction. The carrier structure connecting the TEM-column with 3 bearing points on the frame consists in a stiff tube framework having a lowest Eigenfrequency of above $140 \mathrm{~Hz}$. Coupling is realized by pneumatic damping elements with Eigenfrequencies of $1.8 \mathrm{~Hz}$ laterally and vertically while the column itself possesses a first Eigenmode at clearly above $100 \mathrm{~Hz}$. By this arrangement, one can expect minimized excitation of the eigenmodes of the electron-optical column - obtained by optimized isolation of the column from floor vibrations.

The footprint of the frame measures 1.0 on $1.5 \mathrm{~m}$ and the frontal opening gives enough room for the operator to access the lower part of the column and easily observe the viewing chamber. A view of the CAD-model of the frame is shown in Figure 1.

We report on the experimental realization of the described TEM-column support frame setup and compare the achieved performance with theoretical predictions.

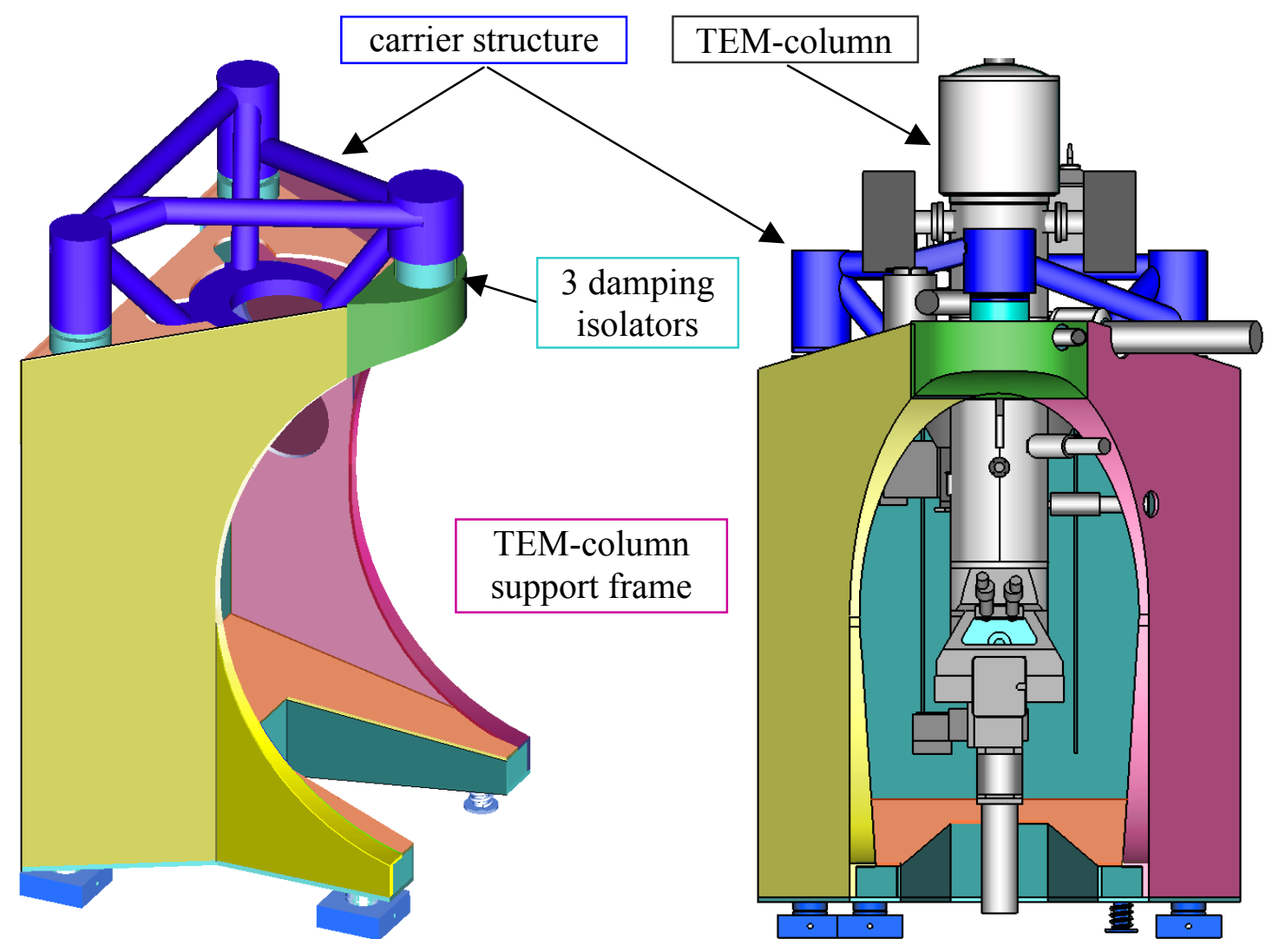

Figure 1: Model view of ultra-stable TEM-column support frame

Corresponding author: Essers@leo.de 